\title{
dspace.vutbr.cz
}

\section{DVB-T2/T2-Lite using MISO Principle for Portable and Mobile Transmission Scenarios}

POLÁK, L.; ŠOTNER, R.; KUFA, J.; KRATOCHVÍL, T.

2021 44th International Conference on Telecommunications and Signal Processing (TSP)

elSBN: 978-1-6654-2933-7

DOl: https://doi.org/10.1109/TSP52935.2021.9522603

Accepted manuscript

C2021 IEEE. Personal use of this material is permitted. Permission from IEEE must be obtained for all other uses, in any current or future media, including reprinting/republishing this material for advertising or promotional purposes, creating new collective works, for resale or redistribution to servers or lists, or reuse of any copyrighted component of this work in other works. POLÁK, L.; ŠOTNER, R.; KUFA, J.; KRATOCHVÍL, T. "DVB-T2/T2-Lite using MISO Principle for Portable and Mobile Transmission Scenarios", 2021 International Conference on Military Technologies (ICMT) - Proceedings, 2021. DOI: 10.1109/TSP52935.2021.9522603. Final version is available at https://ieeexplore.ieee.org/document/9522603 


\title{
DVB-T2/T2-Lite using MISO Principle for Portable and Mobile Transmission Scenarios
}

\author{
Ladislav Polak, Roman Sotner, Jan Kufa and Tomas Kratochvil \\ Brno University of Technology, Faculty of Electrical Engineering and Communication, \\ Department of Radio Electronics, Technicka 12, 61600 Brno, Czech Republic \\ \{polakl, sotner, kufa, kratot\}@feec.vutbr.cz
}

\begin{abstract}
The Second Generation Digital Video Broadcasting Terrestrial (DVB-T2) standard and its light version (DVB-T2Lite) offer very flexible system configurations including multipleinput single-output (MISO) transmission technology to improve the performance of the Single Frequency Networks (SFNs). In this paper, a measurement-based study of the DVB-T2/T2-Lite MISO performance under different mobile and portable transmission scenarios is presented. The outputs of the study, evaluated by both objective and subjective ways, show that the influence of different mobile and portable transmission conditions on the performance of DVB-T2/T2-Lite MISO broadcasting is not negligible.
\end{abstract}

Keywords-DVB-T2, DVB-T2-Lite, MISO transmission, power imbalance, interference, fading, RF measurement, BER, QEF

\section{INTRODUCTION}

The Second Generation Digital Video Broadcasting Terrestrial (DVB-T2) standard and its light version DVB-T2-Lite [1], especially developed for mobile TV broadcasting, have a number of new features in the signal processing chain. For instance, it is possible to mention different scattered pilot patterns (PP), the support of rotated constellation and multipleinput single-output (MISO) transmission mode [2]-[4]. In this work, the MISO-based DVB-T2/T2-Lite broadcasting is in the spotlight. In general, it can improve coverage in Single Frequency Networks (SFNs) [3] as well as ruggedness of the DVB-T2/T2-Lite RF signal under demanding reception conditions [5], [6].

In previous years, attention was mainly devoted to the development of appropriate DVB-T2 MISO configurations for fixed transmission scenarios [7]-[11]. Only several works have focused on the employing of DVB-T2/T2-Lite MISO configuration for mobile and portable TV broadcasting [12] [16]. Tormos et al. [12] introduced a hybrid laboratory/field measurement-based performance comparison of SISO and MISO principles for DVB-T2 mobile reception. It was shown that MISO configuration can be less effective when the receiver (RX) is not optimized for mobile reception. The aim of simulation-based studies [13] and [14] was to find the key factors influencing the quality of DVB-T2 MISO mobile TV signal. Power imbalances between the transmitters (TXs), delays between the diversity components, network density and antenna system configuration have notable impact on the performance of a MISO-based DVB-T2 mobile network.

This work was supported by the BUT internal project no. FEKT-S-20-6325.
Polak et al. [15] explored the DVB-T2-Lite profile using SISO and MISO techniques for broadcasting of mobile TV services. Laboratory measurements, in which Rural Area (RA6) and Bad Urban (BU6) channel models were used, shown connections between the DVB-T2-Lite MISO performance and power imbalance (between TXs) and the considered transmission conditions. The outputs of a trial-based DVB-T2 MISO measurement were published in [16]. It was revealed that the correlation of the signals in an SFN network with a large size is rather low.

Contribution: The aim of this contribution is to provide a laboratory-based exploring of the DVB-T2 and T2-Lite systems utilizing transmit diversity (MISO) for mobile and portable transmission scenarios. Different propagation channel characteristics, emulated by Typical Urban (TU6), Personal Indoor and Outdoor (PI and PO) fading channel models extended by Additive White Gaussian Noise (AWGN) channel model [17], and set of power imbalances for the transmission paths are considered. The performance evaluation of the DVBT2/T2-Lite MISO system is twofold. Firstly, objective parameters, represented mainly by Bit and Modulation Error Ratio (BER and MER), are used to evaluate the TV signal. Secondly, the picture quality of the received TV signal is assessed subjectively. This work is a direct continuation of our previous one [11], in which we have focused on the performance study of DVB-T2 SFN-MISO networks for fixed transmission scenarios. Compared to [9], [15], there are considered different settings of power imbalances between TXs (special transmission conditions), channel models which were not used previously in DVB-T2/T2-Lite MISO performances studies and the objective-based results are completed by subjective ones. The outputs of our contribution extend previously presented works [7], [11], [14], [15] by performance study of DVB-T2Lite using MISO under special transmission configurations. They can be also helpful at the planning of MISO-based SFNs adopted by advanced broadcasting systems [18].

The rest of this paper is organized as follows. The MISO scheme employed by DVB-T2/T2-Lite (see Fig. 1) is briefly introduced in Section II. The used measurement setup and methodology are briefly described in Section III. Sections IV and $\mathrm{V}$ contain the evaluation of the obtained results and conclusion remarks, respectively. 


\section{MISO SCHEME FOR DVB-T2/T2-LITE}

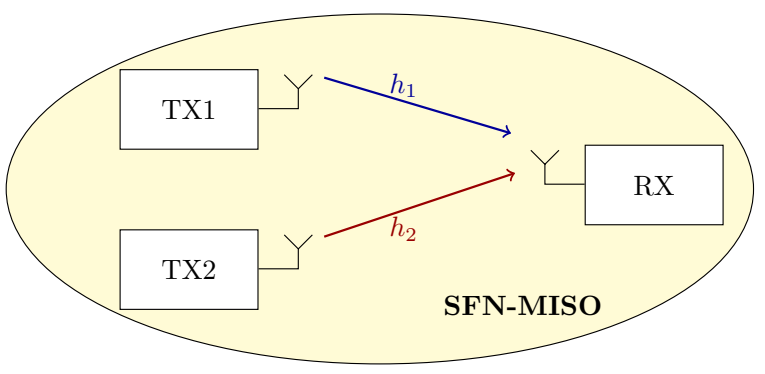

Fig. 1: MISO technique for DVB-T2/T2-Lite.

Transmission mode MISO has been adapted by system DVB-T2/T2-Lite with purpose to improve the gain of SFNs as well as to increase their immunity against echoes due to employing different TXs. The MISO scheme, which consists of at least two TX and one RX (see Fig. 1), introduces space diversity for DVB-T2/T2-Lite and based on the modified Alamouti coding [3]. In this case, symbols (also called as cells) between TX1 and RX (transmission path $h_{1}$ ) are in unchanged form, e.g. $\left[\begin{array}{llll}s_{1} & s_{2} & s_{3} & s_{4}\end{array}\right]$. However, between TX2 and RX (path $h_{2}$ ) are broadcasted in changed form $\left[\begin{array}{llll}s_{2}^{*} & -s_{1}^{*} & s_{4}^{*} & -s_{3}^{*}\end{array}\right]$. As a result, the correlation between the signals radiated by TX1 and TX2 is suppressed. The overlapping of RF signals at the RX's antenna does not cause interference and deep fadings in the RF spectra [19].

\section{Measurement Setup}

The basic concept of the laboratory-based measurement setup used to measure different DVB-T2/T2-Lite MISO transmission scenarios is shown in Fig. 2. Compared to our previously introduced concept [11], [15], this one allows continuous evaluation of a DVB-T2/T2-Lite MISO signal by objective and subjective approaches.

The DVB-T2/T2-Lite MISO RF signal is generated with two broadcast testers, namely SFU and SFE from Rohde\&Schwarz (R\&S), representing transmitters TX1 and TX2. The broadcast tester SFE must be fully synchronized with the SFU to broadcast the MISO signal correctly [11].
TABLE I: DVB-T2/T2-Lite MISO system configurations

\begin{tabular}{|l|c|c|}
\hline Parameters & DVB-T2 & DVB-T2-Lite \\
\hline Code rate (FEC) & $2 / 3$ & $1 / 2$ \\
\hline FECFRAME length & 64800 bits & 16200 bits \\
\hline Modulation & 64-QAM (rotated) & 16-QAM (rotated) \\
\hline FFT mode & $16 \mathrm{~K}$ & $4 \mathrm{~K}$ \\
\hline Carrier mode & extended & normal \\
\hline Guard Interval (GI) & $1 / 16$ & $1 / 8$ \\
\hline Pilot Pattern (PP) & PP3 1 \\
\hline Bandwidth [MHz] & 8 & 8 \\
\hline Channel model & AWGN, PI, PO & AWGN, RA6, TU6 \\
\hline
\end{tabular}

Based on [19], the internal modulator interface (T2-MI) generator of SFU, providing synchronization signals (T2-MI and $1 \mathrm{pps}$ ) and a $10 \mathrm{MHz}$ reference clock, is used to ensure this.

An appropriate video transport stream (TS), according to the defined system parameters (see Table I), is generated in the SFU unit. The SFE broadcast tester uses the same TS as SFU. In the next step, the power level of RF signals $(-40 \mathrm{dBm})$ and channel models in both broadcast testers are set. At the end, both signals are RF modulated $(514 \mathrm{MHz})$ and combined. According to the considered transmission scenarios, transmission conditions (channel models), values of power imbalances (denoted as $\Delta P$ ) and Carrier-to-Noise Ratio $(C / N)$ are accordingly changed during the measurement.

The DVB-T2/T2-Lite MISO RF signal is measured and analyzed by objective and subjective way. The ETL TV analyzer, behind monitoring the spectrum of the RF signal, measures Bit Error Ratio (BER) before and after Forward Error Correction (FEC) decoding, Modulation Error Ratio (MER) and the number of repeated channel decoding (iterations) per FEC Frame (FECFRAME) [3]. The TV signal is processed in the Thomson THT712 set-top-box (STB) and the picture is displayed by a TV. Its quality is evaluated subjectively according to the condition for Quasi-Error Free (QEF) reception [3].

\section{Measurement Results}

Three different transmission scenarios are studied in this work. The first one, so called reference scenario, is represented by three cases. In the first case, AWGN channel conditions are assumed for both $h_{1}$ and $h_{2}$ transmission paths.

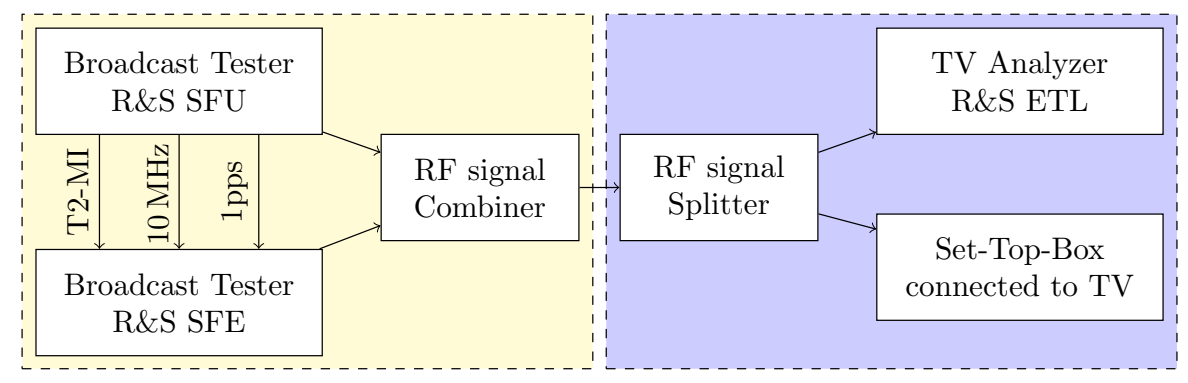

Fig. 2: Measurement of the DVB-T2/T2-Lite MISO signal. Generation of the DVB-T2/T2-Lite MISO RF signal (the TX side) is marked by the yellow dashed block while its measurement (the RX side) is marked by the blue dashed block. The TEROZ $\mathrm{T} 226 \mathrm{~K}$ power combiner is used to combine the RF signals. 


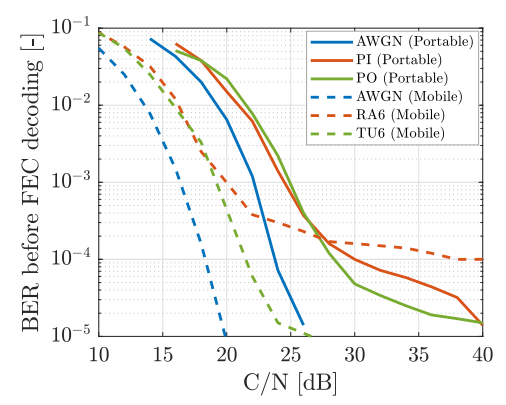

(a) $\mathrm{BER}=\mathrm{f}(C / N)$

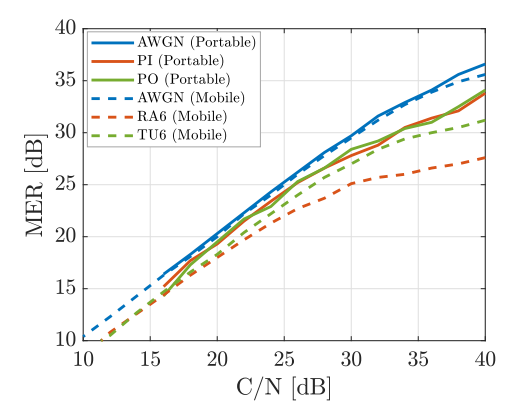

(b) $\mathrm{MER}=\mathrm{f}(C / N)$

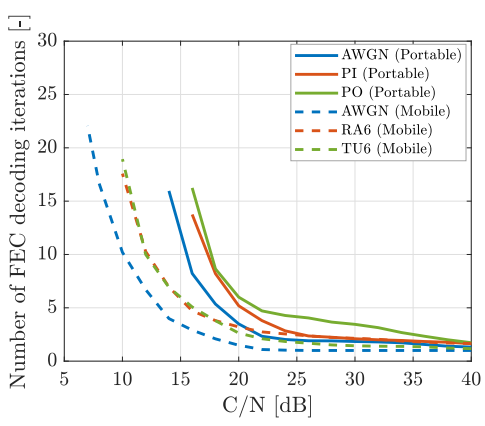

(c) Number of FEC decoding

Fig. 3: Performance of DVB-T2/T2-Lite MISO system for portable and mobile transmission scenarios at $\Delta P=0 \mathrm{~dB}$.

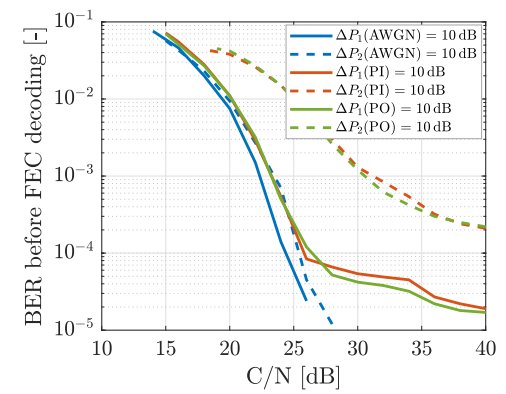

(a) $\mathrm{BER}=\mathrm{f}(C / N)$

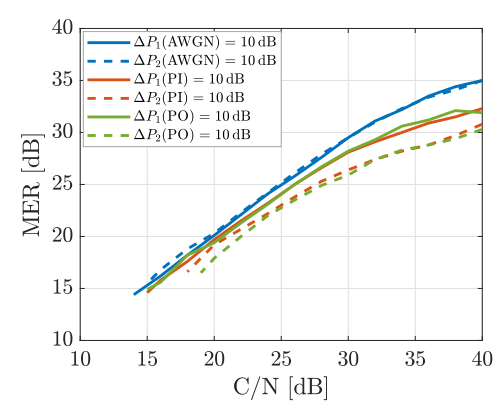

(b) $\mathrm{MER}=\mathrm{f}(C / N)$

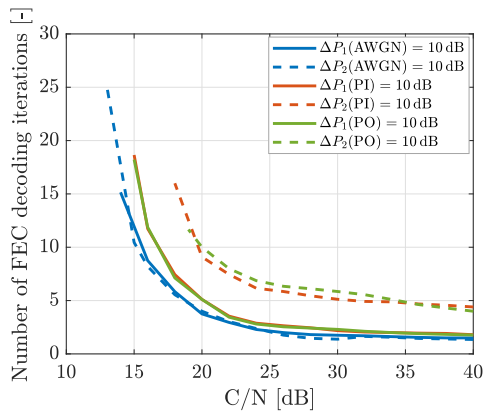

(c) Number of FEC decoding

Fig. 4: Performance of DVB-T2 MISO system for portable transmission scenarios at $\Delta P_{1}=10 \mathrm{~dB}$ and $\Delta P_{2}=10 \mathrm{~dB}$.

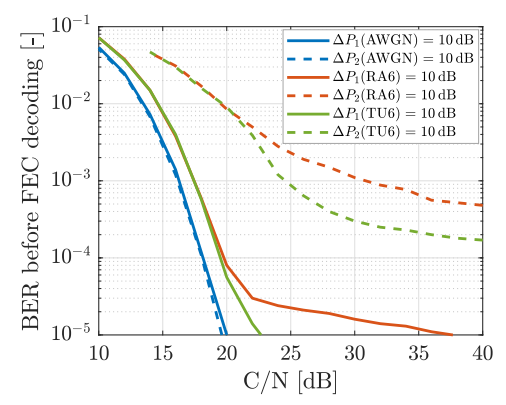

(a) $\mathrm{BER}=\mathrm{f}(C / N)$

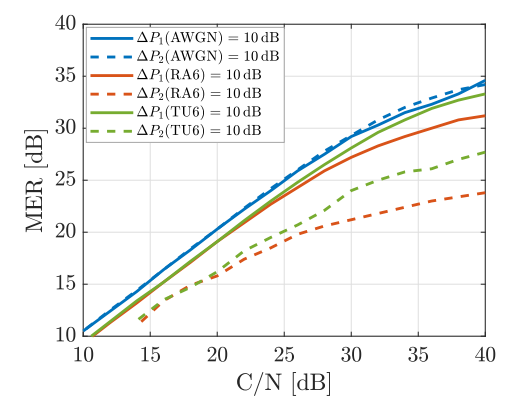

(b) $\mathrm{MER}=\mathrm{f}(C / N)$

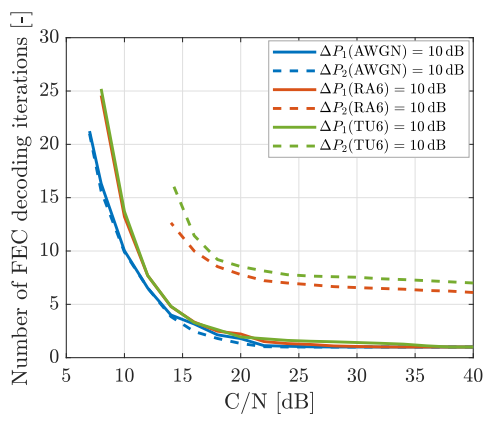

(c) Number of FEC decoding

Fig. 5: Performance of DVB-T2-Lite MISO system for mobile transmission scenarios at $\Delta P_{1}=10 \mathrm{~dB}$ and $\Delta P_{2}=10 \mathrm{~dB}$.

In the second case, path $h_{1}$ represents transmission conditions for portable reception (e.g. in an office) that are emulated by PI and PO fading channel models, while path $h_{2}$ still has the features of the AWGN channel. Finally, in the third case, path $h_{1}$ have the features of RA6 and TU6 mobile fading channel models. In all these cases, we assume $\Delta P=0 \mathrm{~dB}$.

In the second transmission scenario, portable reception of the DVB-T2 MISO signal at power imbalances $\Delta P_{1}=10 \mathrm{~dB}$ and $\Delta P_{2}=10 \mathrm{~dB}$ is considered. At $\Delta P_{1}=10 \mathrm{~dB}$, the signal at the output of TX2 and TX1 has a constant power level of $-40 \mathrm{dBm}$ and $-50 \mathrm{dBm}$, respectively. At $\Delta P_{2}=10 \mathrm{~dB}$, the signal at the output of TX1 and TX2 has a constant power level of $-40 \mathrm{dBm}$ and $-50 \mathrm{dBm}$, respectively.
The third transmission scenario is similar to the second one, but here we focus on the mobile reception of the DVB-T2-Lite MISO signal.

Results from the measurements are shown in Figs. 3-6. It is important to note that the values of $C / N$ are changed simultaneously on both broadcast testers. The so called reference curves $(\Delta P=0 \mathrm{~dB})$ for AWGN, portable and mobile transmission scenarios are plotted in Fig. 3. Thanks to the strong AWGN path and condition $\Delta P=0 \mathrm{~dB}$, lower amount of errors can be achieved for all cases of transmission scenarios at $C / N \geq 24 \mathrm{~dB}$.

The performance of the DVB-T2 MISO signal for portable transmission and defined power imbalances is shown in Fig. 4. 


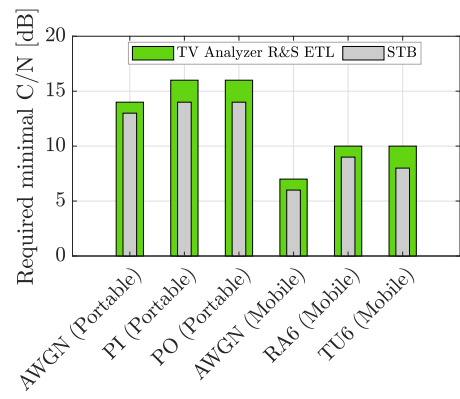

(a) First transmission scenario

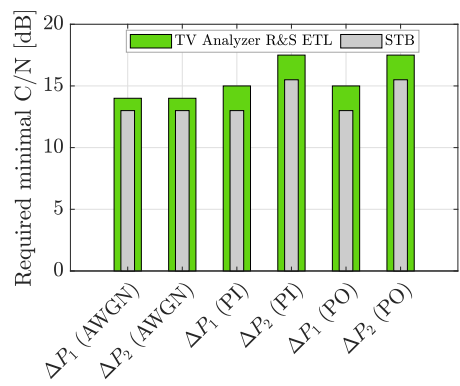

(b) Second transmission scenario

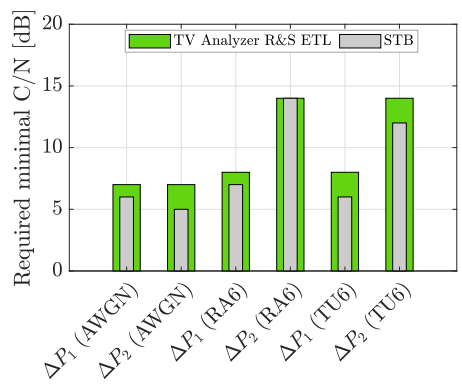

(c) Third transmission scenario

Fig. 6: Required $C / N$ for QEF reception of a DVB-T2/T2-Lite MISO signal related to Fig. 3, Fig. 4 and Fig. 5.

At $\Delta P_{2}=10 \mathrm{~dB}$, the MISO gain for PI and PO fading channels is highly reduced. In comparison with Fig. 3, the BER for $\Delta P_{1}=10 \mathrm{~dB}$ is lower, which is caused by the high dominance of path $h_{2}$ with features of AWGN.

Results from the measurement of the third transmission scenario (mobile reception of the DVB-T2-Lite MISO signal) are captured in Fig. 5. As it is visible, the behavior of the DVB-T2-Lite and DVB-T2 MISO signals (see Fig. 4) is similar thanks to robust T2-Lite system configuration. Once again, $\Delta P_{2}=10 \mathrm{~dB}$ causes higher noises in the transmission environment. In comparison with the portable reception, the number of FEC decoding iterations per FECFRAME at higher $C / N$ values is slightly higher. It is probably caused the RA6 and TU6 fading channel models representing more difficult transmission conditions (e.g. higher speed of the movement of a receiver [17]).

Comparison of the minimum $C / N$ values required to achieve conditions for QEF reception (BER after FEC decoding $\leq 10^{-7}$ ) at the RX, obtained by objective (measured by ETL TV analyzer) and subjective (watching the picture quality on TV) approaches, is shown in Fig. 6. In most cases, the difference between the objective and subjective-based values is very low. Slightly better gain for results related to subjective approach can be explained by better sensitivity of the STB and its advanced channel equalization technique.

\section{Conclusion}

The performance of the DVB-T/T2-Lite system using MISO transmission technique for portable and mobile transmission scenarios was studied. The results confirmed that the performance of DVB-T2/T2-Lite MISO transmission is better when one of the transmission paths have strong features of AWGN channel. However, this gain is notably decreasing, when power level of the signal in this path is decreasing (see the $C / N$ values for $\Delta P_{2}$ in Fig. 6). From the viewpoint of DVB-T2Lite MISO performance, the influence of harder transmission conditions seems to be still minimal when the signal has lower power level in the AWGN-based transmission path than in the path with fading channel characteristics.

\section{ACKNOWLEDGMENT}

The authors would like to thank Vojtech Barta for his help with the laboratory measurements.

\section{REFERENCES}

[1] M. El-Hajjar and L. Hanzo, "A Survey of Digital Television Broadcast Transmission Techniques," IEEE Commun. Surveys \& Tutorials, vol. 15, no. 4, pp. 1924-1949, Fourth Quarter 2013.

[2] ITU-R BT.2254-3, "Frequency and network planning aspects of DVBT2," ITU, Geneva, CH, Report, Aug. 2017.

[3] W. Fischer, Digital video and audio broadcasting technology: a practical engineering guide. Springer Science \& Business Media, 4th ed. 2020.

[4] D. Perez-Calderon and et al., "Simplified metrics calculation for soft bit detection in DVB-T2," Radioengineering, vol. 23, no. 1, pp. 399-404, Apr. 2014.

[5] B. Ruckveratham and S. Promwong., "Empirical single frequency network threshold for DVB-T2 based on laboratory experiments," Turkish Journal of Electrical Engineering \& Computer Sciences, vol. 27, no. 5, pp. 3342-3355, Jan. 2019.

[6] T. Ijiguchi and et al., "Performance Evaluation of Coded SFN for an Advanced ISDB-T," in Proc. of Int. Symp. BMSB, 2020, pp. 1-6.

[7] J. Morgade and et al., "SFN-SISO and SFN-MISO gain performance analysis for DVB-T2 network planning," IEEE Trans. on Broadcast., vol. 60, no. 2, pp. 272-286, June 2014.

[8] S. Jeon, J. Kim, H.-K. Mok and J.-S. Seo, "Formulating the net gain of MISO-SFN in the presence of self-interferences," Radioengineering, vol. 24, no. 2, pp. 643-649, June 2015.

[9] L. Polak, O. Kaller and T. Kratochvil, "SISO/MISO performances in DVB-T2 and fixed TV channels," in Proc. of 38th Int. Conf. on Telecommunications and Signal Processing (TSP), 2015, pp. 768-771.

[10] S. Nepal and et al., "A Comparative Study on Radio Coverage Due to DVB-T2 Multi-Frequency Network (MFN) and Single Frequency Network (SFN)," in Proc. of Int. Symp. BMSB, 2020, pp. 1-6.

[11] L. Polak and et. al, "On the Performance of DVB-T2 MISO System: Special Fixed Transmission Scenarios," in Proc. of 31st Int. Conf. on Radioelektronika, 2021, pp. 1-4.

[12] M. Tormos and et al., "Experimental performance of mobile DVB-T2 in SFN and distributed MISO network," in Proc. of 19th Int. Conf. on Telecommunications (ICT), 2012, pp. 1-5.

[13] N. Cornillet and et al., "Performance of the DVB-T2 system in a Single Frequency Network: Analysis of the distributed Alamouti scheme," in Proc. of Int. Symp. BMSB, 2011, pp. 1-4.

[14] J. Arenas and et al., "Evaluation of the distributed MISO gain for mobile broadcast planning in real scenarios," in Proc. of BMSB, 2012, pp. 1-6.

[15] L. Polak, O. Kaller and T. Kratochvil, "DVB-T2-Lite profile using diversity technique under different channel conditions," in Proc. of Int. Symp. BMSB, 2015, pp. 1-4.

[16] J. Qi and et al., "DVB-T2 MISO field measurements and a calibrated coverage gain predictor," in Proc. of Int. Symp. BMSB, 2012, pp. 1-6.

[17] L. Polak and T. Kratochvil, "Simulation and measurement of the transmission distortions of the digital television DVB-T/H part 3: Transmission in fading channels," Radioengineering, vol. 19, no. 4, pp. $703-711$, Dec. 2010

[18] S. LoPresto and et al., "Transmit Diversity Code Filter Sets (TDCFSs), an MISO Antenna Frequency Predistortion Scheme for ATSC 3.0," IEEE Trans. on Broadcast., vol. 62, no. 1, pp. 271-280, March 2016.

[19] Rohde\&Schwarz Application Note 7BM802E, "Generating a Test Signal for Distributed DVB-T2 MISO,” R\&S, Application Note, Nov. 2013. 